\title{
Estructura y productividad de una población silvestre de Physaria mendocina (Brassicaceae). Implicancias para su desarrollo como cultivo oleaginoso
}

\author{
Wanda J. Masnatta ${ }^{\varpi}$; Luciana GonzÁlez-Paleo \& Damián A. Ravetta \\ CONICET-Museo Egidio Feruglio, Dto. Plantas Actuales. Trelew, Chubut, Argentina.
}

\begin{abstract}
Resumen. Physaria mendocina, es una hierba perenne nativa del Monte en Argentina, de la que existen pocas poblaciones conspicuas y que ha sido propuesta como un nuevo cultivo oleaginoso para zonas áridas. Evaluamos una población en el Parque Nacional Lihué Calel (La Pampa, Argentina) con los objetivos de: 1) caracterizar la distribución espacial, la densidad y la estructura de tamaños, y 2) determinar cómo se relacionan estos factores con la producción de semilla. Se esperaba una distribución agregada con parches de alta y baja densidad. Se evaluó la población durante tres años y se estudiaron las relaciones entre las variables demográficas y las productivas. La población de P. mendocina mostró un patrón de distribución agregado. En parches de menor densidad, aumentó la proporción de individuos de mayor tamaño, en contraposición a los parches más densos, donde prevalecieron individuos más pequeños. Las plantas de menor tamaño tuvieron menor acumulación de biomasa y producción de semilla, jerarquizando la asignación de biomasa hacia estructuras subterráneas. La biomasa individual es el componente que mejor explicó la variación del rendimiento por planta entre individuos de tamaño diferente. El entendimiento de los mecanismos funcionales determinantes de la producción de semillas es un requisito para la conservación y la domesticación de esta especie.
\end{abstract}

[Palabras clave: conservación, hierbas perennes, rendimiento, Patagonia, nuevos cultivos]

\begin{abstract}
Aвstract. Population structure and seed yield of a wild stand of Physaria mendocina (Brassicaceae). Implications for its development as an oilseed crop: Wild stands of the perennial forb Physaria mendocina (syn Lesquerella mendocina, Brassicaceae), are uncommon, small and inconspicuous. This species, native to the Monte desert, is being evaluated as a new oil-seed crop for arid lands. We evaluated a wild population in the Lihué Calel National Park (La Pampa, Argentina) for three years: 2000, 2001, and 2002. The aims of our study were: 1) to characterize the spatial distribution, density and size structure of this population, and 2) to analyze the relationship between population structure and individual plant seed-production. The relationship between population structure and productivity was evaluated using path analysis. We found an aggregated spatial distribution pattern, with patches of high and low density. The proportion of larger size individuals increased in low density patches, respect to those in with high density in which prevailed smaller individuals. Smaller plants had less biomass accumulation and seed production, and had a greater below ground allocation, in relation to larger plants. Individual total biomass was the main determinant of the observed variation in seed-yield in relations to plant size, while harvest index was very stable across the range of plant diameter. The understanding of functional mechanics of seed production should aid in the conservation of wild stands, and in the domestication of this perennial species.
\end{abstract}

[Keywords: conservation, perennial forbs, seed-yield, Patagonia, new crops]

\section{INTRODUCCIÓN}

El género Physaria (syn Lesquerella, Brassicaceae) comprende especies herbáceas nativas de zonas áridas y semiáridas de América. Varias especies del género son consideradas buenos candidatos para el desarrollo de cultivos oleaginosos ya que cumplen con una serie de requisitos que justifican su domesticación (McLaughlin 1985; Ravetta \& Soriano 1998): 1) tienen requerimientos bajos de agua (Dierig etal.1993; Puppala et al. 2004), 2) son tolerantes al estrés hídrico (Ploschuk et al. 2001; González-Paleo

CONICET-Museo Egidio Feruglio, Dto. Plantas Actuales. Av. Fontana 140, (9100) Trelew, Chubut, Argentina.

wmasnatta@mef.org.ar
\& Ravetta 2011b), 3) producen un aceite de alto valor por la presencia de ácidos grasos del grupo hidroxi (Roetheli et al. 1991), y 4) tienen mayor potencial de rendimiento en ambientes semiáridos que en otros ambientes (Ravetta \& Soriano 1998). Del conjunto de 100 especies descritas en este género, Physaria fendleri, $P$. gracilis, $P$. angustifolia (anuales), P. mendocina y P. pinetorum (perennes) han sido elegidas por su potencial agronómico y su tolerancia a la sequía para la introducción en programas de domesticación y selección para zonas áridas (Dierig et al. 1993; Ploschuk et al. 2003, 2005; Windauer et al. 2004; González-Paleo \&

Recibido: 25 de enero de 2012; Fin de arbitraje: 21 de abril; Revisión recibida: 1 de junio; Aceptado: 24 de junio. 
Ravetta 2011a; Masnatta \& Ravetta 2011). Aun cuando su rendimiento de semilla suele ser menor (Wagoner 1990; Deninson 2009, pero ver González-Paleo \& Ravetta, 2011b por una visión alternativa en Physaria), el desarrollo de cultivos perennes ha sido propuesto como una opción más adecuada que el de los cultivos anuales, si se tiene en cuenta que se puede mejorar el manejo del suelo y la eficiencia del uso de agua y nutrientes, y que -en muchos casos- poseen mayor resistencia a estreses abióticos y a plagas. La mayor eficiencia en el uso de los recursos y el sostenimiento del funcionamiento del ecosistema son metas fundamentales en la búsqueda de una mayor sustentabilidad ecológica y económica en ecosistemas frágiles.

Una de estas especies perennes con potencial como nuevo cultivo es Physaria mendocina (Phil.) Kurtz, nativa de la región fitogeográfica del Monte, en Argentina. Esta especie produce semillas con intervalos anuales (iterópara) desde una roseta vegetativa (Correa 1969; Cabrera 1994). Además, presenta una serie de caracteres morfológicos, fisiológicos y fenológicos que podrían contribuir a generar una mayor estabilidad del rendimiento y tolerancia a la sequía, en relación a las especies anuales del mismo género (Ploschuk et al. 2001; González-Paleo \& Ravetta 2011a; González-Paleo \& Ravetta 2011b). Por ejemplo, la biomasa asignada a raíces, la construcción de reservas carbonadas y la morfología foliar, son atributos asociados a la mayor longevidad y tolerancia a la sequía encontrada en $P$. mendocina, cuando se la compara con otras especies anuales y perennes (González-Paleo \& Ravetta 2011a). Sin embargo, se desconoce aún la influencia de la longevidad sobre los caracteres que determinan la producción de semillas, debido a que en la mayoría de los trabajos publicados se han evaluado plantas de hasta dos años de edad, pero no plantas más longevas. En este contexto, las poblaciones naturales presentan una oportunidad valiosa de avanzar en el conocimiento del efecto de la perennidad y la longevidad en los caracteres que modulan el rendimiento, y permite detectar potenciales problemas durante el proceso de domesticación. Además, la evaluación y conservación de la variabilidad de los caracteres de interés productivo, es la base de la mejora genética de los cultivos (Berger et al. 2002).

A pesar de las ventajas que presentan las evaluaciones descriptivas de las poblaciones naturales en las etapas iniciales de la domesticación, las posibilidades son limitadas ya que varias especies del género Physaria están categorizadas como "especies amenazadas" de América (Dierig et al. 2004). Para P. mendocina, aunque de la evaluación de las colecciones de ejemplares de herbario en la Argentina, surge que las poblaciones naturales estaban distribuidas ampliamente en el Monte hasta inicios del siglo XX. En la actualidad son pocas, de tamaño reducido, y apenas visibles. En extensos viajes de colección, encontramos una fracción de aquellas poblaciones citadas y sólo una población importante en el Parque Nacional Lihué Calel (La Pampa, Argentina). En este contexto, existe la necesidad de conservar la variabilidad genética necesaria para asegurar la estabilidad de la especie (sensu Allendorf \& Luikart 2007), para lo cual es necesario comprender la condición demográfica y ecológica de las poblaciones que aún existen de Physaria mendocina.

Uno de los primeros pasos para el manejo sustentable de un recurso vegetal silvestre es determinar la estructura de la población y su producción potencial. Debido a que no existen antecedentes de estudios demográficos de las poblaciones naturales de P. mendocina, se desconocen su estructura de tamaños y su densidad, así como los factores que afectan la estructura poblacional. Si bien el análisis espacial por sí mismo no permite determinar qué proceso está originando la distribución de una población, este estudio puede proporcionar información valiosa relativa a los posibles factores causales y puede contribuir a generar hipótesis sobre los procesos que han sido importantes en su génesis (Pielou 1961; Silvertown \& Wilson 1994).

La distribución espacial de las poblaciones está determinada por numerosos procesos que actúan de manera simultánea; entre esos procesos se encuentran las interacciones competitivas y de facilitación (Callaway 1995; Pacala et al. 1996) que juegan un papel clave en el funcionamiento de los ecosistemas (McAuliffe 1984). La heterogeneidad en la distribución de los recursos del suelo típica de los ecosistemas áridos (Aguiar \& Sala 1999) determina que rara vez las poblaciones se distribuyan de manera aleatoria o regular, $\mathrm{y}$ frecuentemente se encuentran disposiciones agregadas (Legendre \& Fortin 1989). Además, las interacciones planta-planta también determinan el tamaño, el crecimiento, la supervivencia y la productividad de los individuos de la población. Así, en ambientes con baja disponibilidad de agua y nutrientes - 
donde la intensidad de competencia es mayorlas plantas tendrían un patrón de asignación preferencial hacia raíces y una productividad menor (Weiner 2004).

En este contexto, esperamos una distribución agregada de la población silvestre de $P$. mendocina, con parches de alta y baja densidad. En los parches de alta densidad prevalecerán individuos de menor tamaño, que asignarán biomasa de forma preferencial a raíces, mientras que en parches de baja densidad predominarán individuos de tamaño mayor, con producción mayor de semillas. Para poner a prueba estas hipótesis examinamos la población nativa de Physaria mendocina situada en el Parque Nacional Lihue Calel (La Pampa, Argentina) con las siguientes preguntas: (1) ¿cuál es la distribución espacial, la densidad y la estructura en clases de tamaños? y (2) ¿cómo influyen estos tres factores en los caracteres productivos de ésta especie?

\section{MATERIALES y MÉTODOS}

\section{Área de estudio}

El estudio se llevó a cabo durante tres años en el Parque Nacional Lihué Calel, en el centro-sur de la provincia de La Pampa, Argentina. El clima es templado-árido, con una precipitación media anual de $400 \mathrm{~mm}$ y una temperatura media anual que oscila entre 10 y $14^{\circ} \mathrm{C}$ (Cinti 1983). La flora del lugar es una estepa arbustiva abierta dominada por diversas especies de jarilla (Larrea divaricata, L. cuneifolia y L. nitida), Prosopis alpataco, Monthea aphylla y Schinus fasciculatus, una comunidad típica de la provincia fitogeográfica del Monte (Cabrera 1994).

\section{Caracterización de parámetros poblacionales}

Los muestreos se realizaron en el mes de diciembre de los años 2000, 2001 y 2002, momento en el cual las plantas se encuentran con frutos maduros. Se determinó un área general de muestreo de 300×300 $\mathrm{m}$, dentro de la que se delimitó, para cada año, un rectángulo de $2940 \mathrm{~m}^{2}$ (48 m de ancho x $72 \mathrm{~m}$ de largo). En este rectángulo se realizaron cuatro transectas separadas por $12 \mathrm{~m}$. En cada transecta se dispusieron nueve parcelas de $0.8 \mathrm{~m}^{2}$ ( $8 \mathrm{~m}$ de largo x $0.1 \mathrm{~m}$ de ancho) en las que se evaluó la densidad, la estructura por tamaños y la distribución espacial de la población.

La densidad se calculó como el número de individuos por unidad de área, para cada una de las parcelas en cada transecta (para un total de 36 parcelas). Las parcelas fueron clasificadas en: 1-10, $10-20$ y 20-40 plantas $/ \mathrm{m}^{2}$. Para evaluar la estructura por tamaños, los individuos dentro de cada parcela se clasificaron en cuatro clases de diámetro: $\leq 2$, de
2 a 4 , de 4 a 6, y $>6 \mathrm{~cm}$. El diámetro fue calculado tomando un promedio de dos mediciones de la roseta de cada uno de los individuos muestreados. Para cada parcela se determinó la proporción de individuos correspondiente a cada clase diametral. Para determinar el patrón de distribución espacial se contó el número de individuos dentro de cada parcela muestreada. A partir de estos datos de estimó el índice de Morisita (Is; Morisita 1962). Debido a que éste índice indicó que la población presentó un patrón agregado, se evaluó el ajuste de los datos a una distribución binomial negativa utilizando la prueba de bondad de ajuste chicuadrado.

\section{Caracterización de parámetros productivos}

Se muestrearon al azar para cada año un mínimo de 10 plantas para cada clase de diámetro. Las plantas se cosecharon utilizando cilindros de $40 \mathrm{~cm}$ de diámetro y $60 \mathrm{~cm}$ de profundidad, de modo de incorporar la mayoría de las raíces de cada planta. En todos los casos se inspeccionó el borde del suelo extraído y se verificó la ausencia de raíces. El material cosechado fue llevado al laboratorio y la biomasa total fue clasificada en hojas, raíces, frutos y semillas. El rendimiento de cada uno de los individuos se determinó como g de semilla/ planta, y fue desglosado como el producto de sus componentes, la biomasa total por el índice de cosecha, que indica la proporción que representa el peso de las semillas con respecto a la planta entera, incluidas las raíces. El patrón de partición se expresó como una proporción de la biomasa total: proporción de biomasa de hojas (LMR, del inglés "leaf mass ratio") y proporción de biomasa de raíz (RMR, del inglés "root mass ratio").

\section{Análisis de datos}

Evaluamos el efecto de la densidad y el año sobre la proporción de individuos de diferentes clases diamétricas por medio de un ANVA de dos factores: año y densidad. Analizamos las diferencias entre clases diamétricas en los tres años de estudio en cuanto a: la producción de semillas, la biomasa total, el índice de cosecha y el patrón de asignación proporcional (raíces y hojas); este análisis lo realizamos por medio de un ANVA de dos factores: año x diámetro de planta. Los supuestos del ANVA fueron puestos a prueba utilizando la prueba de normalidad de Shapiro-Wilk's y la prueba de homogeneidad de varianzas de Levene. La biomasa total y la proporción de biomasa de raíces fueron transformadas con logaritmo natural de forma de cumplir con dichos supuestos.

Para determinar los efectos directos e indirectos de la estrategia de asignación sobre la producción de semillas entre individuos de diferente clase de diámetro, realizamos un análisis de vías ("path analysis") (Arbuckle \& Wothke 1999). Consideramos un modelo teórico hipotético para explicar cómo las diferencias en el patrón de asignación (proporción de biomasa destinada a 
hojas-LMR, raíces-RMR e índice de cosecha-IC) entre clases diamétricas, influyen de manera directa o indirecta (a través de alterar la biomasa total) en la producción de semillas. Este modelo se generó para los tres años de estudio tomados en conjunto, debido a que los valores de las variables utilizadas entre clases diamétricas no difirieron entre años (interacción año $x$ diámetro no significativa).

\section{Resultados}

\section{Variables poblacionales}

La población presentó un patrón agregado de distribución (Tabla 1). La distribución observada en la población natural no difirió significativamente de la distribución teórica esperada, binomial negativa $(P=0.91 ; P=0.43$; $P=0.15$ para los años 2000, 2001 y 2002, respectivamente) (Figura 1). La interacción entre la densidad y el año fue no significativa $(P>0.05)$, por lo cual el efecto de la densidad sobre la proporción de individuos de diferentes clases diamétricas se evaluó para los tres años en conjunto. Así, los individuos de las clases diamétricas $\leq 2$ y $2-4 \mathrm{~cm}$ aumentaron su proporción de ocurrencia en parches de alta densidad (Figura 2). En contraposición, los individuos de mayor tamaño $(2-4 \mathrm{y}>6 \mathrm{~cm})$ prevalecieron en parches de menor densidad (Figura 2).

\section{Variables productivas}

En ninguna de las variables medidas se encontró interacción año $x$ diámetro $(P>0.05)$, es decir que las diferencias entre clases diamétricas en la biomasa y su partición se mantuvieron durante los tres años de estudio (Tabla 2). Las plantas pequeñas (diámetro $\leq 2$ $\mathrm{cm})$ que crecieron en parches de alta densidad mostraron una mayor asignación de biomasa a raíces a expensas de una menor asignación a hojas, en relación al patrón de partición de plantas de mayor tamaño (diámetro $>6 \mathrm{~cm}$ ) (Tabla 2). Asimismo, las plantas de menor diámetro tuvieron una menor acumulación de biomasa y producción de semillas en relación a las de mayor tamaño. El índice de cosecha

Tabla 1. Análisis del Patrón de dispersión de la población natural Physaria mendocina en el Parque Nacional Lihué Calel, durante tres años de consecutivos.

Table 1. Analysis of the population distribution pattern of wild Physaria mendocina in the National Park Lihue Calel, for three consecutive years.

\begin{tabular}{lccc}
\hline Estadísticos descriptivos & 2000 & 2001 & 2002 \\
\hline Índice de Morisita & 4.24 & 1.68 & 2.26 \\
F crítico & 846.75 & 647.81 & 453.97 \\
Interpretación & Agregado & Agregado & Agregado \\
\hline
\end{tabular}

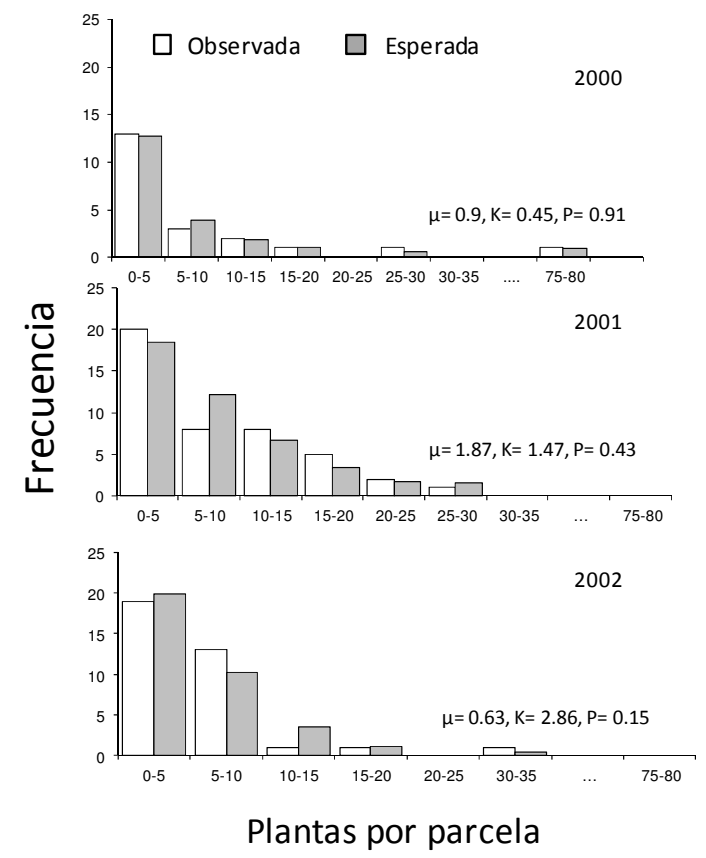

Figura 1. Distribución de frecuencias esperadas y observadas para una población natural de $P$. mendocina durante tres años consecutivos comparada con una distribución binomial negativa. Se presentan los parámetros $\mu, \mathrm{k}$ y el valor $P$ de la distribución estadística.

Figure 1. Observed and expected (binomial negative) frequency distribution for a wild population of $P$. mendocina for three years. We showed the parameters $\mu, \mathrm{k}$ and $P$ value for adjust of the statistical distribution.

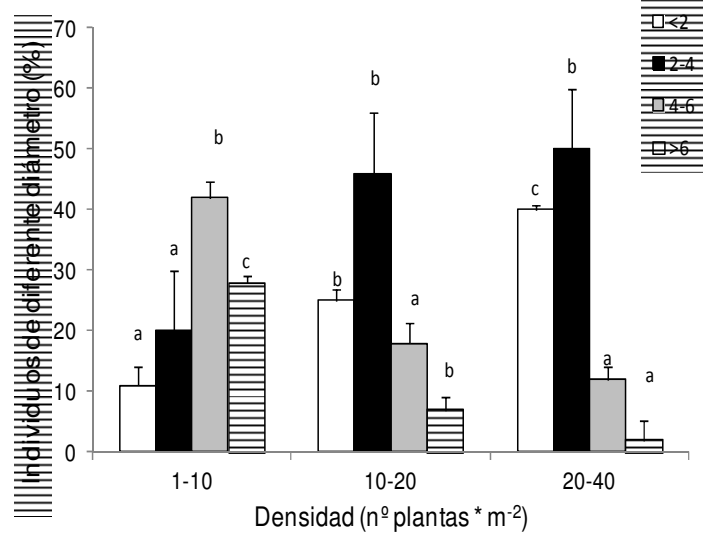

Figura 2. Proporción de individuos pertenecientes a diferentes clases diamétricas en función de la densidad, para la población natural de $P$. mendocina evaluada durante tres años consecutivos. Letras distintas indican diferencias significativas entre densidades para una misma clase diamétrica $(P<0.05)$. Las barras verticales indican el error estándar.

Figure 2. Proportion of individuals of each diameter class as function of density for P. mendocina growing in a wild population in Lihue Calel. Different letters indicate significant differences between densities for the same diameter class $(P<0.05)$. Vertical bars indicate the standard error. 
Tabla 2. Patrón de asignación de biomasa en función del tamaño de las plantas para la población nativa de Physaria mendocina. Valores medios y resultados de los ANOVA para probar la significancia $(P<0.05)$ para cada unos de los tamaños. IC=proporción de biomasa destinada a semilla; LMR=proporción de biomasa destinada a hojas; RMR=proporción de biomasa destinada a raíz. Se muestran Los valores del F crítico (F) los grados de libertad (GL) y el valor $P$ de significancia $(P)$.

Table 2. Results of the two way ANOVA for comparison of seed-yield, total biomass, harvest index and allocation pattern (RMR-root mass ratio and LMR-leaf mass ratio) for each diameter class for the wild population of Physaria mendocina. F-values, degrees of freedom (GL) and $P$ values are showed.

\begin{tabular}{|c|c|c|c|c|c|}
\hline Diámetro & Producción de semillas (g) & $\mathrm{IC}(\%)$ & Biomasa total $(\mathrm{g})$ & RMR (\%) & LMR (\%) \\
\hline$<2$ & $0.02 \pm 0.004 \mathrm{a}$ & $4.7 \pm 0.8 \mathrm{~ns}$ & $0.46 \pm 0.05 a$ & $23 \pm 2 b$ & $60 \pm 2 a$ \\
\hline $2-4$ & $0.07 \pm 0.01 \mathrm{a}$ & $6.2 \pm 0.7 \mathrm{~ns}$ & $1.33 \pm 0.19 b$ & $18 \pm 1 \mathrm{a}$ & $62 \pm 1 \mathrm{a}$ \\
\hline $4-6$ & $0.18 \pm 0.02 b$ & $6.8 \pm 0.8 \mathrm{~ns}$ & $2.55 \pm 0.19 c$ & $15 \pm 1 \mathrm{a}$ & $66 \pm 2 b$ \\
\hline$>6$ & $0.38 \pm 0.05 c$ & $5 \pm 0.9 \mathrm{~ns}$ & $6.49 \pm 0.68 \mathrm{~d}$ & $18 \pm 2 \mathrm{a}$ & $66 \pm 2 b$ \\
\hline $\mathrm{F}$ & 58.55 & 1.36 & 89.27 & 6.12 & 3.13 \\
\hline GL & 132 & 132 & 132 & 132 & 132 \\
\hline$P$ & 0.001 & 0.26 & 0.001 & 0.001 & 0.028 \\
\hline
\end{tabular}

fue similar para las distintas clases diamétricas (Tabla 2).

\section{Mecanismos funcionales asociados a la variación de la productividad}

Encontramos un buen ajuste de los datos al modelo puesto a prueba mediante el análisis de vías $\left(\chi^{2}=1.45 ; P=0.31\right)$. La diferencia en la producción de semillas entre individuos de diferente diámetro creciendo en parches de alta y baja densidad estuvo relacionada con el patrón de asignación aéreo y subterráneo (relación negativa entre LMR y RMR, y

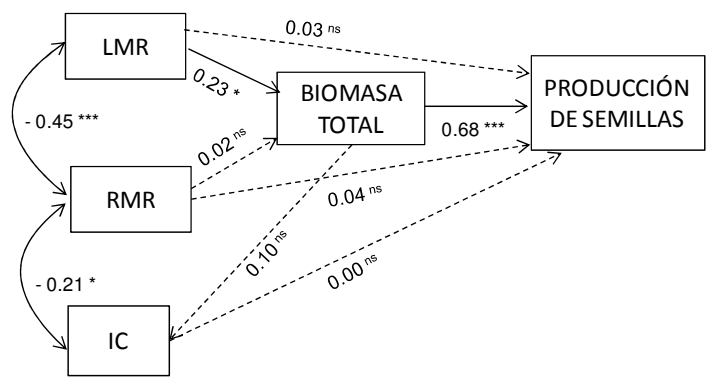

Figura 3. Análisis de vías (path analysis) representando los efectos directos e indirectos del patrón de asignación de biomasa (LMR: proporción de biomasa destinada a hojas, RMR: proporción de biomasa destinada a raíces, IC: índice de cosecha, y biomasa total) sobre las diferencias en la producción de semillas entre individuos de diferentes diámetros de P. mendocina. Cada flecha posee su coeficiente estandarizado de vía y su probabilidad $\left({ }^{*} P<0.05 ;{ }^{* *} P<0.01,{ }^{* * *} P<0.001\right)$. Flechas continuas indican relaciones significativas, flechas punteadas indican relaciones no significativas.

Figure 3. Path analysis showing the direct and indirect effects of allocation pattern to leaf (leaf mass ratio, LMR) and to root (root mass ratio, RMR), harvest index, and total biomass on differences in seed production between individuals of different diameters class of $P$. mendocina. Each arrow has its standardized path coefficient and its probability $\left({ }^{*} P<0.0\right.$ $\left.5,{ }^{* *} P<0.01,{ }^{* * *} P<0.001\right)$. Solid arrows indicate significant relationships, while dashed arrows indicate no significant relationships. relación positiva entre LMR y biomasa total) (Figura 3). El efecto del patrón de asignación sobre la producción de semillas fue indirecto, a través de cambios en la biomasa total (relación positiva con la producción de semilla). La relación entre la producción de semillas y el índice de cosecha no fue significativa (Figura 3).

\section{DisCUSIÓN}

En los tres años analizados la población de Physaria mendocina de Lihué Calel presentó un patrón de distribución agregado (Figura 1). Las causas de la agregación pueden ser diversas. En particular para algunas especies del género Physaria, se ha propuesto que los factores que afectan la distribución espacial serían las características del microambiente, los mecanismos de dispersión de semillas, los sitios apropiados para la germinación, los patrones de disturbio natural, y las interacciones planta-planta (Cabin \& Marshall 2000; Cabin et al. 2000). En P. flenderi se encontró que la gran mayoría de las semillas permanecen en un radio no mayor a un metro desde de la planta parental (Cabin 1996). La dispersión a corta distancia explicaría la tendencia de los individuos más jóvenes a la formación de manchones (Ehrlen \& Eriksson 2000; Matteucci et al. 1979). Sin embargo, el mismo patrón agregado puede ser generado por diferentes factores, por lo cual su génesis es difícil de dilucidar (Schurr et al. 2004). Como ya fue mencionado, las interacciones plantaplanta como la competencia y facilitación, son factores que pueden determinar el patrón de distribución en la vegetación de zonas áridas (Aguiar et al. 1992; Fowler 1988). Así, la distribución agregada encontrada para la población de $P$. mendocina podría surgir del efecto conjunto de la interacción positiva entre plántulas y la planta adulta, y la posterior competencia. 
Los mecanismos implicados en la facilitación son diversos, e incluyen la creación de un microclima favorable y protección frente a herbívoros, entre otros (Callaway 1995; Callaway \& Pugnaire 1999). Si bien dicha interacción se produce en una amplia variedad de ambientes (Callaway 1995), su importancia aumenta en zonas áridas y semiáridas, debido a que permite amortiguar los efectos del estrés ambiental (e.g., Nobel 1984; Bush \& Van Auken 1991). En particular en ambientes áridos, la protección de la planta madre mejora el establecimiento de las plántulas y asegura la incorporación de nuevos individuos a la población (Flores \& Jurado 2003).

Por otra parte, la competencia por los recursos del suelo es un proceso generalizado en las plantas de zonas áridas (Manning \& Barbour 1988; Briones et al. 1996). Entre los factores que regulan la intensidad de la competencia, la densidad es uno de los principales. En este sentido, en la población natural que evaluamos, aunque la densidad se mantuvo estable durante los tres años de estudio, al analizarla en función del tamaño de las plantas, encontramos que en los parches de mayor densidad predominaron los individuos pequeños(Figura 2). El tamaño de un individuo adulto en general refleja la disponibilidad global de recursos (van Noordwijk \& de Jong 1986). Así, un aumento en la densidad de los parches llevaría a una mayor competencia y como consecuencia de la reducción en la cantidad de recursos disponibles, las plantas serían de menor tamaño (Casper 1996). Para la población de $P$. mendocina el aumento del número de individuos pequeños asociado al incremento de la densidad podría reflejar la competencia intraespecífica.

La competencia es uno de los factores que puede determinar el patrón de partición y la productividad de los individuos de una población. En la población estudiada, las plantas de menor tamaño creciendo en parches de alta densidad asignaron más biomasa a raíces a expensas de una menor asignación a hojas, en relación a las de mayor tamaño. Esta diferencia en el patrón de asignación resultó en una menor acumulación de biomasa y producción de semilla de las plantas de parches densos (Tabla 2 y Figura 3). La asociación entre la densidad y el patrón de partición está de acuerdo con lo esperado según la teoría de asignación óptima (Bloom et al. 1985). Para este caso, en las plantas de parches de alta densidad la prioridad de asignación a raíces podría resultar en un aumento de la capacidad de adquisición de los recursos del suelo. En cuanto a la reducción de la productividad en $P$. mendocina, es de esperar que este cambio en el patrón de asignación de individuos afectados por la competencia tenga consecuencias negativas sobre la dinámica y la persistencia de la población, tanto en el corto como en el largo plazo (Eriksson \& Ehrlén 1992; Lennartsson 2002, para otros casos de estudio). La disminución en la producción de semillas es una de las amenazas principales para los procesos de la historia de vida que afectan de forma directa la estructura de edades de la población (Lennartsson 2002). En conclusión, proponemos que la distribución espacial agregada de la población natural de Physaria mendocina sería consecuencia del patrón de dispersión de semillas, de interacciones positivas primero y negativas una vez que las plantas están establecidas, y determinaría la estructura en clases de tamaño. Esta propuesta deberá ponerse a prueba con experimentos manipulativos, pero permite pre-diseñar prácticas de manejo adecuadas para el establecimiento del cultivo de $P$. mendocina.

En términos del desarrollo de nuevos cultivos, la comprensión de los procesos de facilitación contribuiría a la determinación de los requerimientosóptimosparaelestablecimiento de las plantas y su supervivencia en estadios tempranos del cultivo. En este sentido, en trabajos de resiembra natural realizados en parcelas de cultivo experimental de $P$. pinetorum se identificó al estadio de plántula como la etapa más vulnerable del proceso de reclutamiento (Bar-Lamas 2010). Así, a través de la facilitación de las plántulas reclutadas por parte de las plantas adultas, y la posterior auto-regulación denso-dependiente del número de plantas, se establecería la estructura final del siguiente stand de cultivo, con plantas de diferente edad. La competencia al final del ciclo de cultivo podría generar efectos negativos en la productividad (biomasa y semillas) con densidades equivalentes a cincuenta mil plantas por hectárea (parches de mayor densidad). En contraste, para la especie comercial de ciclo anual $P$. fendleri, se ha propuesto que no hay efecto de la competencia intraespecífica sobre el rendimiento y sus componentes hasta densidades de hasta un millón de plantas por hectárea (Brahim et al. 1998).

Por otro lado, al analizar la generación del rendimiento a través del análisis de vías se observó que la producción de semillas de los 
individuos en parches de alta y baja densidad fue explicada por los cambios en la biomasa total y no por el índice de cosecha (Figura 3). En términos agronómicos, el incremento en la producción potencial puede alcanzarse de dos formas: incrementando la producción total de materia seca o incrementando la proporción que es destinada a los órganos cosechables. En cuanto al índice de cosecha, los valores documentados para $P$. mendocina en este trabajo oscilan entre 4 y $7 \%$, mientras que para la especie comercial $P$. fendleri, en condiciones de cultivo y con adecuado suministro de agua y nutrientes, los valores varían entre 8 y 14\% (Brahim et al. 1998). Aunque la diferencia en el índice de cosecha entre la especie comercial y $P$. mendocina fue de sólo $50 \%$, la diferencia del rendimiento es de al menos un orden de magnitud (entre 0.02 y $0.38 \mathrm{~g} /$ planta en $P$. mendocina y 2.08 y $3.97 \mathrm{~g} /$ planta en $P$. fendleri). Se desprende de este análisis que la biomasa total ha sido el carácter de mayor cambio para explicar el aumento del rendimiento en el proceso de selección en Physaria. Este incremento en la biomasa ha sido acompañado por un aumento enorme de la demanda de agua del cultivo, con la consecuente necesidad de riego (800 a 1000 $\mathrm{mm}$ de agua total es requerida para completar el ciclo de cultivo en las siembras comerciales) (Thompson et al. 1989). El aumento del tamaño de las plantas podría implicar otros cambios adicionales al aumento del consumo. Entre nuestros resultados encontramos que el aumento de la biomasa total de la planta produjo también cambios en el patrón de asignación: las plantas de mayor biomasa tuvieron mayor proporción de biomasa aérea, a expensas de una menor proporción de raíz (Figura 3). Esta estrategia de partición podría generar un balance negativo entre la absorción de agua por las raíces y la transpiración por las hojas. La susceptibilidad de la planta al estrés hídrico aumentaría en las etapas finales del cultivo, cuando se esperan los déficits hídricos en un clima de tipo mediterráneo como el de la Patagonia. Asimismo, la menor proporción de raíz podría comprometer la habilidad de perpetuación y reducir la longevidad. En conjunto, estos resultados permitirían generar un marco teórico a partir del cual se elijan los criterios de selección y se pongan a prueba estrategias de manejo adecuadas para la introducción de $P$. mendocina al cultivo en zonas áridas.

Agradecimientos: Agradecemos a G. Cerdeiras por el apoyo en las tareas de campo y laboratorio. Este trabajo se realizó con financiamiento económico del Fondo Nacional de Ciencia y Técnica, FONCyT PID 363.

\section{BiBLIOGRAFÍA}

Aguiar, MR \& OE Sala. 1999. Patch structure, dynamics and implications for the functioning of arid ecosystems. TREE, 14:273-277.

Aguiar, MR; A Soriano \& O Sala. 1992. Competition and facilitation in the recruitment of seedlings in the Patagonian steppe. Funct. Ecol., 6:66-70.

Allendorf, FW \& G Luikart. 2007. Conservation and the Genetics of Populations. Wiley-Blackwell Publishing. Pp. 642. Second edition.

ArbuCKLE, JL \& W WothKe. 1999. AMOS. 4.0 User`s Guide. SPSS. (SmallWalters, Chicago).

BÄr-Lamas, M. 2010. Reclutamiento voluntario en especies del género Physaria. Posible utilización como método de resiembra en ambientes de bajos recursos. Tesis de Licenciatura, Universidad Nacional de la Patagonia San Juan Bosco. Trelew, Chubut-Argentina.

BERGER, JD; LD ROBERTSON \& PS COCKS. 2002 Agricultural potential of Mediterranean grain and forage legumes: Key differences between and within Vicia species in terms of phenology, yield, and agronomy give insight into plant adaptation to semi-arid environments. Genet. Resour. Crop Ev. 49:313-325.

Bloom, AJ; FS Chapin \& HA Mooney. 1985. Resource limitation in plants-an economic analogy. Annu. Rev. Ecol. Syst., 16:363-392.

Brahim, K; DT RAY \& DA Dierig. 1998. Growth and yield characteristics of Lesquerella fendleri as a function of plant density. Ind. Crops Prod., 9:63-71.

Briones, O; C Montaña \& E Escurra. 1996. Competition between three chihuahuan desert species: evidence from plant size-distance relations and root distribution. J. Veg. Sci., 7:453-460.

Bush, JK \& OW Van AuKen. 1991. Importance of time of germination and soil depth on growth of Prosopis glandulosa (Leguminosae) seedlings in the presence of a C-4 grass. Am. J. Bot., 78:1732-1739.

Cabin, RJ \& DL Marshall. 2000. The demographic role of soil seed banks. Spatial and temporal comparisons of below-and above-ground populations of the desert mustard Lesquerella fendleri. J. Ecol., 88:283-292.

CABIN, RJ. 1996. Genetic comparisons of seed bank and seedling populations of perennial desert mustard, Lesquerella fendleri. Evolution, 50:1830-1841.

Cabin, RJ; DL Marshall \& RJ Mitchell. 2000. The demographic role of soil seed banks. II. Investigations of the fate of experimental seeds of the desert mustard Lesquerella fendleri. J. Ecol., 88:293-302.

Cabrera, A. 1994. Regiones fitogeográficas Argentinas. Enciclopedia Argentina de Agricultura y Jardinería. Tomo II. Pp. 85. Fascículo 1. Acme S.A.C.I.

Callaway, RM \& FI Pugnaire. 1999. Facilitation in communities. Pp. 623-648 en: Pugnaire, FI \& F Valladares (eds.). Handbook of Functional Plant Ecology. Marcel Dekker, Inc., Nueva York, Estados Unidos.

Callaway, RM. 1995. Positive interactions among plant. The Botanical Review, 61:306-349.

CASPER, BB. 1996. Demographic consequences of drought in the herbaceous perennial Cryptantha ava: effects of density, associations with shrubs, and plant size. Oecologia, 106:144-152.

CinTI, RR. 1983. Parque Nacional Lihué Calel. Aire y Sol (ed.). Abril S.A., Bs. As. Administración de Parques Nacionales.

Correa, MN. 1969. Flora Patagónica (Argentina). Typhaceae a 
Orquidaceae. Tomo VIII. Parte III. Colección Científica del INTA. Buenos Aires. Pp. 219.

DENINSON, RF. 2009. Darwinian Agriculture: real, Imaginary and Complex Trade-offs as Constraints and Opportunities. Pp. 215-233 en: Sadras, V \& D Calderini (eds.). Crop Physiology: applications for genetics improvements and agronomy. Academic Press.

Dierig, DA; AE Thomson \& FS NAKAYAMA.1993. Lesquerella commercialization efforts in the United States. Ind. Crops Prod., 1:289-293.

DIERIG, D; MP TOMASI; AM SALYWON \& DT RAY. 2004. Improvement in hydroxy fatty acid seed oil content and other traits from interspecific hybrids of three Lesquerella species: Lesquerella fendleri, L. pallida, and L. lindheimeri. Euph., 139:199-206.

EHRLÉN, J \& O ERIKSSON. 2000. Dispersal limitation and patch occupancy in forest herbs. Ecology, 81:1667-1674.

ERIKSSON, O \& J EHRLÉN. 1992. Seed and microsite limitation of recruitment in plant population. Oecologia, 91:360364.

Flores, J \& E JuRADO. 2003. Are nurse-protégé interactions more common among plants from arid environments? J. Veg. Sci., 14:911-916.

FowLER, NL. 1988. What is a safe site?: Neighbour, litter germination date, and patch effects. Ecology, 69:947-961.

GonZÁlez-PALEO, L \& DA RAVEtTa. 2011a. Indirect changes associated with a selection program for increased seedyield in wild species of Lesquerella (Brassicaceae): Are we developing a phenotype opposite to the expected ideotype? Ind. Crops Prod., 34:1372-1380.

GonzÁlez-Paleo, L \& DA RavetTa. 2011b. Relationships between reproductive output, morpho-physiological traits and life span in Lesquerella (Brassicaceae) Ind. Crops Prod., 34:1386-1392.

Legendre, P \& MJ Fortin. 1989. Spatial pattern and ecological analysis. Vegetatio, 80:107-138.

LENNARTSSON, T. 2002. Extinction thresholds and disrupted plant-pollinator interactions in fragmented plant populations. Ecology, 83:3060-3072.

MANNING, SJ \& DP BARBOUR. 1988. Root systems, spatial patterns and competition for soil moisture between two desert subshrubs. Am. J. Bot., 75:885-893.

Masnatta, WJ \& DA Ravetta. 2011. Seed-yield and yield components response to source-sink ratio in annual and perennial species of Lesquerella (Brassicaceae). Ind. Crops Prod., 34:1393-1398.

Matteucci, SD; A Colma \& L Pla. 1979. Análisis de la Vegetación y el Ambiente del Estado Falcón; La vegetación. Publicaciones del Dto. de Investigación del Inst. Univ. Tecnol. de Coro, Venezuela. Pp. 292.

McAuliffe, JR. 1984. Saguaro-nurse tree associations in the Sonora desert: competitive effects of saguaros. Oecologia, 64:319-321.

MCLAUGHLIN, SP. 1985. Economic prospects for new crops in the southwestern United States. Econ. Bot., 39:473-481.

Morisita, M. 1962. Ig-Index, a measure of dispersión of individuals. Res. Popul. Ecol., 5:1-7.

Nobel, PS. 1984. Extreme temperaturas and thermal tolerances for seedlings of desert succulents. Oecologia, 62:310-317.

VAN NoORdwiJK, AJ \& G DE Jong. 1986. Acquisition and allocation of resources: their influence on variation in life history tactics. Am. Nat., 128:137-142.

Pacala, SW; CD Canham; J Saponara; JA Silander; RK KOBE; ET AL. 1996. Forest models defined by field measurements: Estimation, error analysis, and dynamics. Ecol. Monogr., 66:1-43.

PIELOU, EC. 1961. Segregation and symmetry in twospecies populations as studied by nearest neighbor relationships. J. Ecol., 49:255-569.

Ploschum, EL; G Cerdeiras; L Windauer A Dierig \& DA RAVETTA. 2003. Development of alternative Lesquerella species in Patagonia (Argentina): potential of Lesquerella angustifolia. Ind. Crops Prod., 18:1-6.

Ploschuk, EL; GA Slafer \& DA Ravetta. 2005. Reproductive allocation of biomass and nitrogen in annual and perennial Lesquerella crops. Ann. Bot., 96: 127-135.

PloschuK, EL; L Windauer \& DA Ravetta. 2001. Potential Value of traits associated with perennial habit in the development of new oil-seed crops for arid lands. A comparison of Lesquerella fendleri and L. mendocina subjected to water stress. J. Arid. Environ., 47:373-386.

Puppala, N; JL Fowler; TL Jones; L Gutschick \& L Murray. 2004. Evapotranpiration, yield, and water-us efficiency responses of Lesquerella fendleri at different growth stages. Ind. Crops Prod., 21:33-47.

RavetTA, DA \& A SoRiano. 1998. Alternatives for the development of new industrial crops for Patagonia. Ecol. Austral, 8:297-307.

Roetheli, JC; KD Carlson; R Kleiman; AE Thompson; DA DIERIG; ET AL. 1991. Lesquerella as a source of hydroxyl fatty acids for industrial products. USDA-CSRS. Office of Agr. Mat. Growing Industrial Material Series (unnm.). Washington, DC.

Schurr, FM; O Bossdorf; SJ Milto \& J Schumacher. 2004. Spatial pattern formation in semi-arid shrubland: a priori predicted versus observed pattern characteristics. Plant Ecol. 173:271-282.

Silvertown, J \& JB WiLSON. 1994. Community structure in a desert perennial community. Ecology, 75:409-417.

WAGONER, P. 1990. Perennial grain development: Past efforts and potential for future. Crit. Rev. Plant Sci., 9:381-408.

WeINER, J. 2004. Allocation, plasticity, and allometry in plants. Perspect. Plant Ecol. Evol. Syst., 6:207-215.

Windauer, L; GA Slafer \& D Ravetta. 2004. Phenological responses to temperature of an annual and a perennial Lesquerella species. Ann. Bot., 94:139-144. 